\title{
Experimental Analysis Of Carbopol Gels Inside a Cubical Cavity Across Differentially Heated Plates
}

\author{
Kunal Dhananjay Jadhav \\ Dept. of Mechanical, Industrial and \\ Aerospace Engineering \\ Concordia University \\ Montreal, Canada \\ kunalels148@gmail.com
}

\author{
Peter Rossi \\ Dept. of Mechanical, Industrial and \\ Aerospace Engineering \\ Concordia University \\ Montreal, Canada \\ peterrossi79@gmail.com
}

\author{
Ida Karimfazli \\ Dept. of Mechanical, Industrial and \\ Aerospace Engineering \\ Concordia University \\ Montreal, Canada \\ ida.karimfazli@concordia.ca
}

\begin{abstract}
Viscoplastic fluids are the class of fluids with a yield stress that governs their ability to flow. This research deals with the effect of temperature difference applied across a viscoplastic fluid (carbopol gel) and studies the various flow regimes depending on the onset of the flow. Reasonable efforts have been made to estimate the experimental flow onset times and investigate the onset of the flow at different yield stress values. The idea behind the proposed research is to characterize the natural convection of yield stress fluids. The flow field characterization is carried out using a PIV system. The post processing is done using PIVlab, a useful add-on for MATLAB. In this paper, $\Delta \mathrm{T}$ refers to the temperature difference across the cavity. We investigated that noflow $\Delta \mathrm{T}$ is increased as yield stress increases. We also found that flow development is delayed with an increase in yield stress and decreases with increasing $\Delta T$ for all carbopol concentrations.

Index Terms-Natural Convection, Flow Development, Motionless Limit, Flow Onset, 2-D Flow Field
\end{abstract}

\section{INTRODUCTION}

Non-Newtonian fluids are used in our homes for day to day purposes; some commonly used fluids are honey, face cream, and toothpaste. This class of fluids exhibit flow properties different from Newtonian depending on their ability to flow. Also termed as viscoplastic fluids, they have a threshold stress value below which they cease to flow. This threshold stress is termed as yield stress, which is the minimum applied shear stress necessary for the fluid to flow. Viscoplastic fluids exhibit dual modes of the state; that is below the yield stress, they act as a solid body, and above this limit, they flow like a fluid [1]. The following research is an experimental investigation of carbopol gels inside a cubical cavity with differentially heated sidewalls. Flow dynamics work on the principle of temperature-dependent density variation, which results in buoyancy force [3]. The ratio of yield stress to buoyancy force is a dimensionless number called Bingham

$$
B=\tau_{y} /\left(\rho_{o} g \beta \Delta T\right)
$$

where $\tau_{y}, \rho_{o}, \mathrm{~g}, \beta, \Delta \mathrm{T}$ refers to shear stress, density, acceleration due to gravity, coefficient of thermal expansion, and temperature difference across the cell, respectively. The balance between buoyant forces and yield stress determines the flow onset [2]. The flow regime before the onset is governed by conduction and convection after the initiation of onset [4]. Numerical studies of natural convection inside a cubical cavity for viscoplastic fluids have been presented by Karimfazli and Frigaard [2] and Turan, Chakraborty, and Poole [3]. Karimfazli and Frigaard have solved a 2D problem analogous to our experiments. As the domain does not have buoyant forces initially, the flow takes a finite time before it starts [4]. Turan, Chakraborty and Poole have given the flow profile for the vertical velocity component in a 2-D flow field [3]. Kebiche, Castelain, and Burghelea have conducted experiments with carbopol gels to characterize Rayleigh-Bénard convective states [5]. Davaille, Gueslin, Massmeyer, and Di Giuseppe analyzed the thermal instabilities developed in yield stress fluids heated from below. They also measured the occurrence of the convective regime as well as onset time for thermal plumes [6]. To sum up, earlier experimental and theoretical studies on viscoplastic fluids have explored the behavior of these fluids in a thermally induced flow field. This research aims to study the development of convective currents in a square cavity. The effect of yield stress and the temperature difference across the cell have been investigated experimentally. A brief outline of the paper is as follows: experimental setup and methodology are presented in section II. Section II-A describes the selection and preparation of carbopol gels and rheological characterization. Section II-B is dedicated to the experimental setup, data acquisition techniques, and methods. Results are presented in Section III followed by concluding remarks in the final section of the paper.

\section{EXPERIMENTAL SETUP AND Methodology}

\section{A. Selection and preparation of carbopol gels}

Carbopol is a cross-linked polyacrylic acid homopolymer, which is a great thickening agent. Carbopol ETD 2050NF powder is mixed with deionized water using a mechanical mixer at 600 RPM for about 2 hours to ensure homogeneous dispersion. The solution is further neutralized by gradually adding $5 \mathrm{M} \mathrm{NaOH}$ while mixing at $30 \mathrm{RPM}$ until a thick gellike solution is obtained. The mixing speed of the mechanical mixer is kept low at this stage to avoid bubble formation. Carbopol gels of concentration ranging between $0.3 \mathrm{~g} / \mathrm{L}$ to 
$0.4 \mathrm{~g} / \mathrm{L}$ were prepared for experiments. Finally, tracer particles were added after the neutralization was completed.

Rheological characteristics of the samples were measured using a Hybrid DHR-3 TA rheometer with a parallel plate geometry of $20 \mathrm{~mm}$ diameter (flat surface). The results are shown in Fig. 1. The data with shear rates lower than $10^{-2}$ have not been considered for measuring yield stress. The suitable range of shear rate, corresponding to the small velocities in the flow domain, is considered to be $10^{-2} s^{-1}$ to $1 s^{-1}$. HerschelBulkley model was fitted to the data to find the rheological properties. The results are shown in table I. The HerschelBulkley equation is described by

$$
\sigma=\sigma_{o}+K \dot{\gamma}^{n}
$$

where $\sigma$ is the shear stress, $\sigma_{o}$ is the yield stress of the material, $\mathrm{K}$ is the consistency, $\mathrm{n}$ is the flow index and $\dot{\gamma}$ is the strain rate.

\section{B. Experimental setup and data acquisition technique}

We are using two TE modules from TE technology (model no. $\mathrm{CP}-121)$ to maintain the desired temperature on the sidewalls. A TE (thermo-electric) device module works on the principle of the peltier effect. The experiments are performed in a cubical cell of 0.5 inch thick walls made of acrylic (see Fig. 2). The two side walls are made of aluminum plates $1 / 2$ inch thick. These aluminum plates are positioned between the cell wall and TE units to facilitate heat transfer. Thermal pads are used between aluminum plates and the TE units to ensure proper thermal contact for efficient heat transfer. The experimental setup is equipped with two TC48-20 temperature controllers and a USB multifunction data acquisition device from LabJack model U6 pro (DAQ). Fig. 3 shows an exploded view of the experiment setup. There are

TABLE I

RHEOLOGICAL PARAMETER BASED ON STRAIN RATES $\left(10^{-2}-1\right) s^{-1}$

\begin{tabular}{|c|c|c|c|}
\hline Concentration (g/L) & Yield stress (Pa) & $\mathbf{K}\left(\mathbf{P a} s^{n}\right)$ & $\mathbf{n}$ \\
\hline 0.3 & 0.04 & 0.07 & 1.33 \\
\hline 0.35 & 0.07 & 1.12 & 1.06 \\
\hline 0.38 & 0.10 & 1.29 & 1.17 \\
\hline 0.4 & 0.14 & 1.75 & 0.92 \\
\hline
\end{tabular}

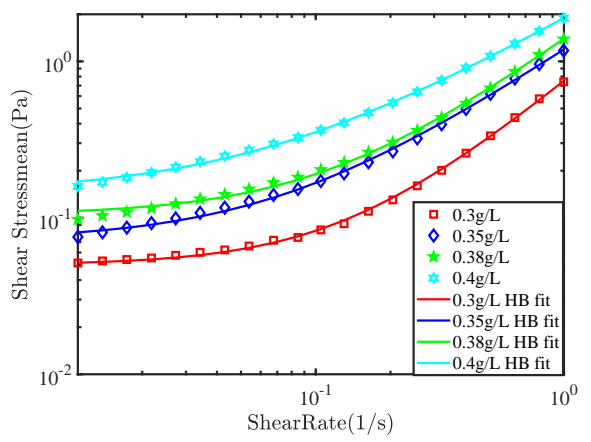

Fig. 1. Rheology comparison with their fits.

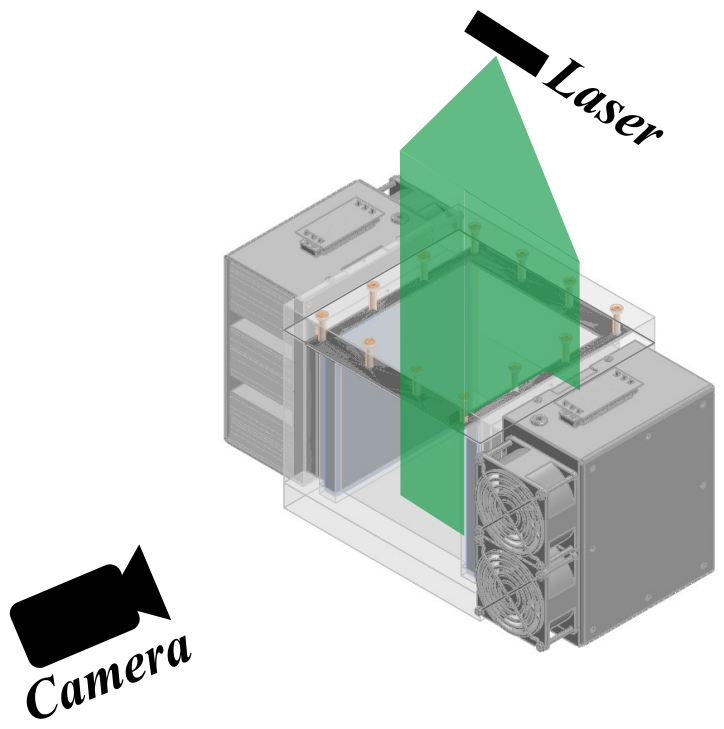

Fig. 2. Experimental Setup.

two thermocouples placed on either side, one at the center of the wall (e.g., see $T \_2$ in Fig. 3) and one on the top corner (e.g., see $T \_5$ in Fig. 3 ). These temperatures are collected throughout the experiment using the DAQ. A thermistor is positioned in the middle of the top surface of each peltier unit (e.g., see $T_{-} 1$ in Fig. 3). These thermistors are connected to the temperature controllers and used to impose the temperature boundary conditions.

Particle image velocimetry (PIV) is used to measure the velocity field throughout the flow development. PIV components used for our experiments were a camera with a synchronization unit and a class 4 laser head. We illuminate a 2-D midplane of the cell using a laser sheet and capture

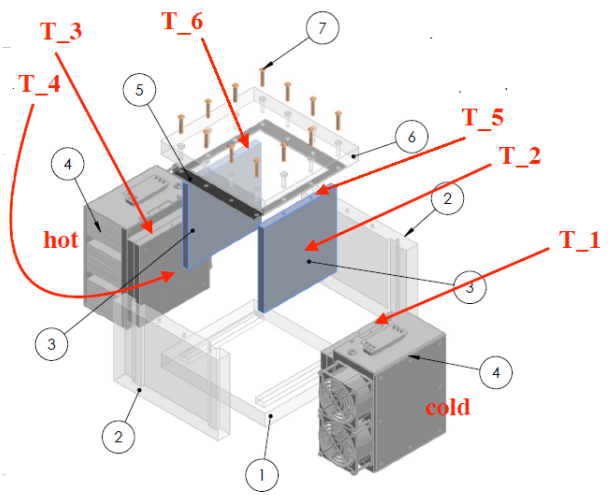

Fig. 3. Components of experimental setup- a) Part No.1- 1 unit of Cast acrylic bottom. b) Part No.2- 2 units of Cast acrylic right-left side of cell . c) Part No.3- 2 units of $1 / 2$ inch thick aluminium plates. d) Part No.4- 2 units of CP121 TE device. e) Part no.5- 1 Silicion rubber gasket. f) Part No.6- 1 unit of Cast acrylic top. g) Part No.7- 12 cap screws of socket button head. 
images throughout the experiment. The acquired images were exported for further post-processing. PIV-lab was used to post-process the experimental image data [7]. Images 20500s apart were correlated for calculating the velocity. All the experiments were carried out against a dark background. The duration of the tests was long enough to ensure the flow regimes achieve a steady state.

\section{RESULTS}

We use the $L^{2}$ norm of the velocity field to analyze fluid flow development. The $L^{2}$ norm of a function is given by

$$
|f|=\left(\int_{X}|f(x)|^{2} d x\right)^{1 / 2}
$$

where $X$ represents the flow domain. We will denote the velocity norm by $\|u\|$ in this paper. We have considered the temperature differences ranging from 5 to $33{ }^{\circ} \mathrm{C}$ for all the concentrations. Please note that in this paper $\Delta t_{c c o r r}$ and $\Delta \mathrm{T}$ denote the cross-correlation time between image pairs and temperature difference between the plates, respectively.

\section{A. Velocity norm and temperature evolution}

Fig. 4 illustrates the flow and temperature evolution. As the Peltier units are turned on, the temperatures on the plates undergo transition before reaching the steady state set temperatures. This transition of temperatures on the aluminum plates is faster than the flow evolution to steady state.

\section{B. Evolution of the velocity field}

Figs. 5(b-d) are the velocity magnitude colormaps given at three different instances of flow development. The three red dots in Fig. 5a represent three separate instances of the flow development. The first dot corresponds to the onset of flow $(t=340 s)$, illustrates the velocity field when the velocity norm is maximum $(t=1830 \mathrm{~s})$, and the last dot illustrates the steady state $(t=10730 s)$. It can be seen that flow develops more rapidly on the hot wall. This is expected as the heating power of Peltier units is higher than their cooling power.

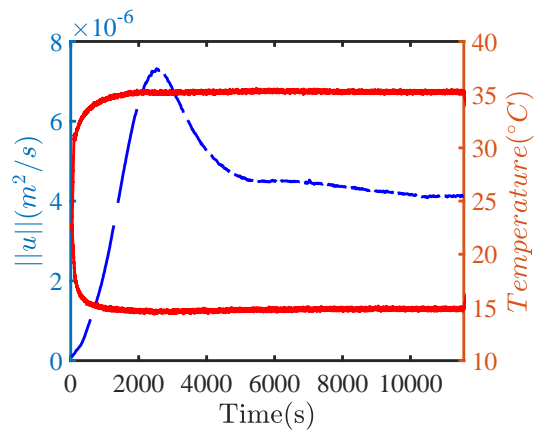

Fig. 4. Temperature and Norm Evolution, $\Delta t_{c c o r r}=20 \mathrm{~s}, 0.3 \mathrm{~g} / \mathrm{L}, \Delta \mathrm{T}=23^{\circ} \mathrm{C}$.
TABLE II

ONSET TIMES

\begin{tabular}{|c|c|c|}
\hline Concentration(g/L) & $\Delta \mathbf{T}\left({ }^{\circ} \mathrm{C}\right)$ & $t_{\text {onset }}(\mathbf{s})$ \\
\hline 0.3 & 33 & 320 \\
\hline & 28 & 340 \\
\hline & 23 & 520 \\
\hline & 15 & 701 \\
\hline & 33 & 717 \\
\hline 0.35 & 28 & 739 \\
\hline & 23 & 1116 \\
\hline & 15 & 1972 \\
\hline & 5 & No flow \\
\hline & 33 & 1191 \\
\hline 0.38 & 15 & No flow \\
\hline & 33 & No flow \\
\hline 0.4 & \multicolumn{2}{|c}{}
\end{tabular}

\section{Flow onset}

The theoretical studies predict that the flow onset should be delayed by a finite time; but experimentally we observe an immediate flow onset after the peltier units were turned on (see Fig. 6). To compare the timescale of the initial development of the flow, we define the onset times as time velocity norm takes to reach $12 \%$ of its maximum value. Flow onset times of the experiments are tabulated in Table II. Fig. 8a shows that $t_{\text {onset }}$ decreases with increasing temperature differences for all carbopol concentrations. Fig. 8b shows the relationship between $t_{\text {onset }}$ and concentration for $\Delta \mathrm{T}=33^{\circ} \mathrm{C}$. It is seen that $t_{\text {onset }}$ increases as concentration is increased.

\section{Flow Development}

Figs. 7(a-d) show the development of flow throughout the experiment in samples of different concentrations and subject to different $\Delta T$. Flow development curves suggest that flow

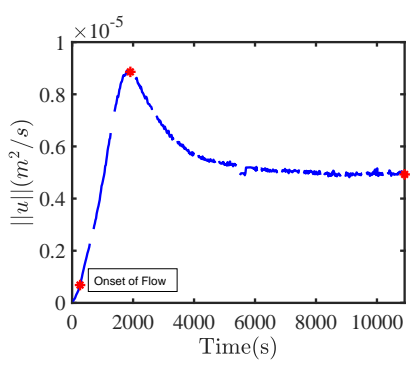

(a) $\Delta t_{c c o r r}=20 \mathrm{~s}, 0.3 \mathrm{~g} / \mathrm{L}$.

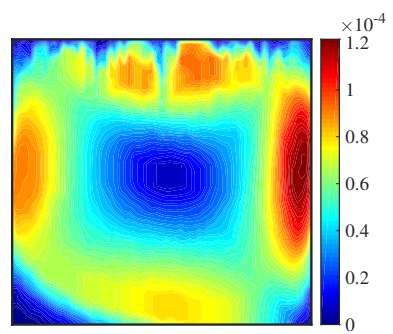

(c) At $t=1830 \mathrm{~s}$ (peak).

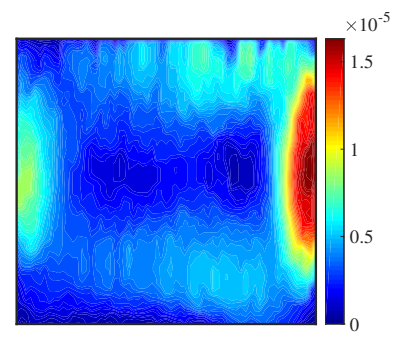

(b) At $t=340 \mathrm{~s}$ (onset).

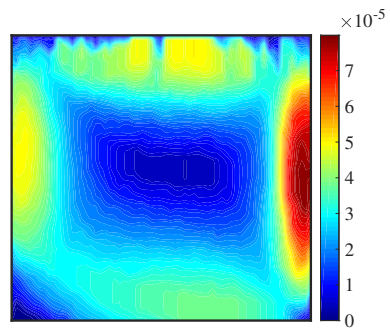

(d) At $t=10730 \mathrm{~s}$ (steady).
Fig. 5. Snapshots of evolution of velocity field. 


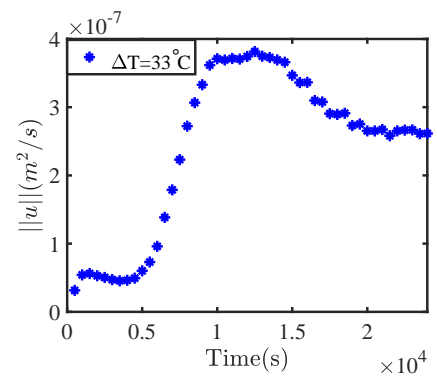

Fig. 6. $\Delta t_{c c o r r}=500 \mathrm{~s}, 0.38 \mathrm{~g} / \mathrm{L}$.

initially accelerates before decelerating and approaching the final steady state. Fig. 7(a-d), also illustrate that the maximum velocity of the flow decreases with decreasing $\Delta T$ and increasing the yield stress. It has also been observed that at sufficiently low $\Delta T$, there is no steady flow; see Fig. 7(b-d).

Repeatability tests were conducted for all carbopol concentrations to ensure repeatability of the results. The error bars in Figs. 9(a-d) show the standard deviation of the instantaneous velocity norm. The standard error of mean for $90 \%$ of the experimental data points was found to be less than $10 \%$, and the standard error of mean for remaining data points was less than $20 \%$ for all the carbopol concentrations.

\section{E. The motionless limit}

The motionless limit refers to the maximum $\Delta \mathrm{T}$ for a given working fluid below which there is no measurable steady flow. Fig. 8c illustrates that the critical $\Delta \mathrm{T}$, below which steady flow is not observed, increases with the concentration.

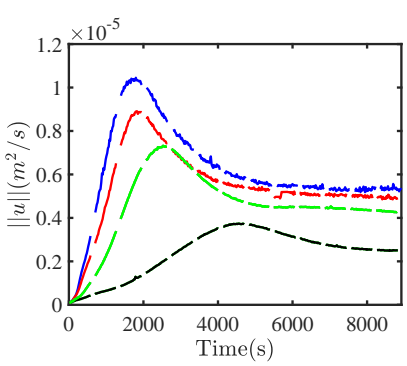

(a) $\Delta t_{\text {ccorr }}=20 \mathrm{~s}, 0.3 \mathrm{~g} / \mathrm{L}$.

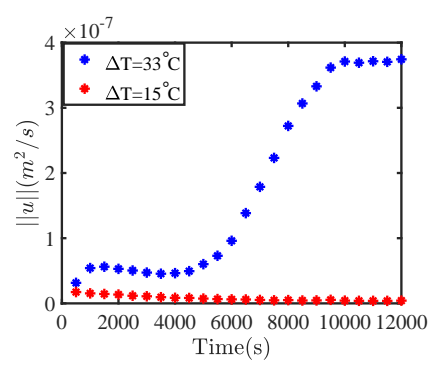

(c) $\Delta t_{\text {ccorr }}=500 \mathrm{~s}, 0.38 \mathrm{~g} / \mathrm{L}$.

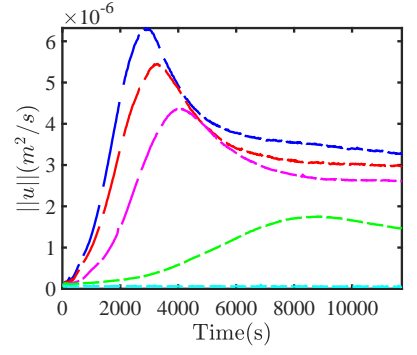

(b) $\Delta t_{\text {ccorr }}=20 \mathrm{~s}, 0.35 \mathrm{~g} / \mathrm{L}$.

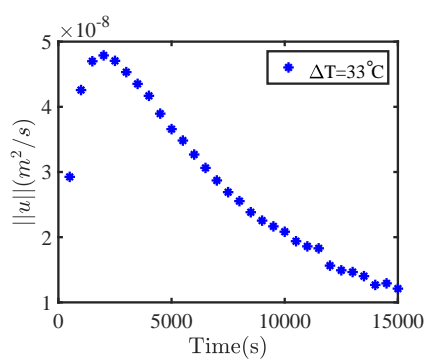

(d) $\Delta t_{\text {ccorr }}=500 \mathrm{~s}, 0.4 \mathrm{~g} / \mathrm{L}$.
Fig. 7. Figs. 7(a-d) are flow development curves a) Blue- $\Delta \mathrm{T}=33^{\circ} \mathrm{C}$, Red$\Delta \mathrm{T}=28^{\circ} \mathrm{C}$, Green- $\Delta \mathrm{T}=23^{\circ} \mathrm{C}$, Black- $\Delta \mathrm{T}=15^{\circ} \mathrm{C}$. b) Blue- $\Delta \mathrm{T}=33^{\circ} \mathrm{C}$, Red$\Delta \mathrm{T}=28^{\circ} C$, Magenta- $\Delta \mathrm{T}=23^{\circ} C$, Green- $\Delta \mathrm{T}=15^{\circ} C$, Cyan- $\Delta \mathrm{T}=5^{\circ} C$.

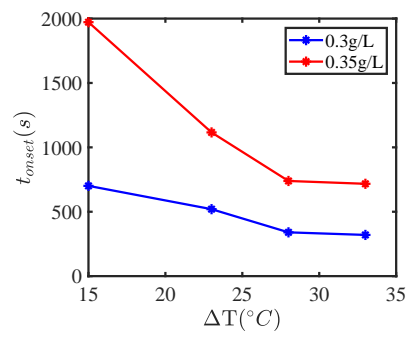

(a)

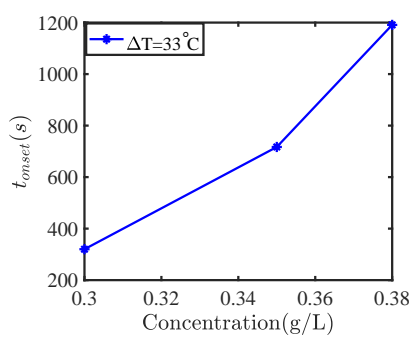

(b)

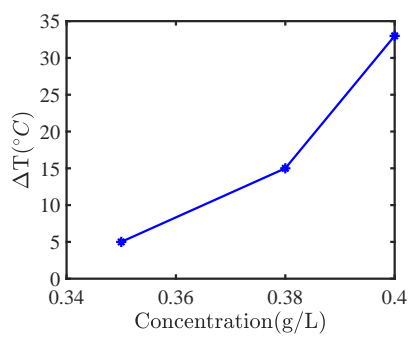

(c)

Fig. 8. a) $t_{\text {onset }}(s)$ vs $\Delta \mathrm{T}\left({ }^{\circ} C\right)$. b) $t_{\text {onset }}(s)$ vs Concentration $(\mathrm{g} / \mathrm{L})$. c) Motionless limit $\left(\Delta \mathrm{T}\left({ }^{\circ} C\right)\right)$ vs Concentration $(\mathrm{g} / \mathrm{L})$.

\section{CONCLUding REMARKS}

To conclude, this study illustrates the relationship between flow development and parameters like yield stress and temperature difference. It has been shown that the evolution of the velocity field occurs on the hot side of the cell first because of the higher heating capacity of TE device. It has been observed

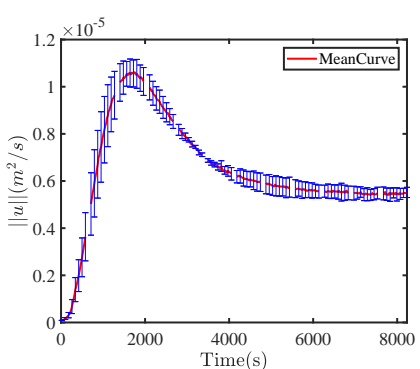

(a)

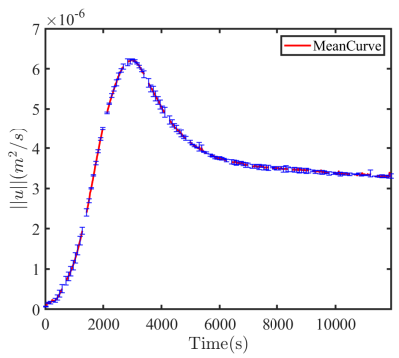

(c)

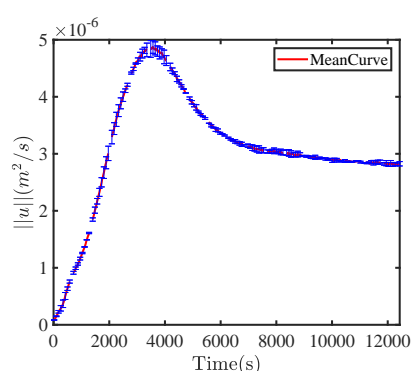

(b)

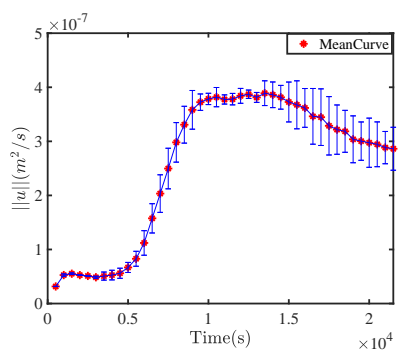

(d)
Fig. 9. a) Error bar for $0.3 \mathrm{~g} / \mathrm{L}, \Delta \mathrm{T}=33^{\circ} \mathrm{C}$ b) Error bar for $0.3 \mathrm{~g} / \mathrm{L}, \Delta \mathrm{T}=15^{\circ} \mathrm{C}$ c) Error bar for $0.35 \mathrm{~g} / \mathrm{L}, \Delta \mathrm{T}=33^{\circ} \mathrm{C}$ d) Error bar for $0.38 \mathrm{~g} / \mathrm{L}, \Delta \mathrm{T}=33^{\circ} \mathrm{C}$ 
that maximum flow speed is reduced with increasing carbopol concentrations. It has also been shown that maximum critical $\Delta \mathrm{T}$, below which there is no steady flow, increases with yield stress. We also found that $t_{\text {onset }}$ increases with yield stress and decreases with $\Delta \mathrm{T}$ for all carbopol concentrations.

\section{REFERENCES}

[1] F. Irgens. Rheology and non-newtonian fluids. Springer, 2014.

[2] I. Karimfazli, IA Frigaard, and Anthony Wachs. A novel heat transfer switch using the yield stress. Journal of Fluid Mechanics, 783:526-566, 2015.

[3] O. Turan, N. Chakraborty, and R.J. Poole. Laminar natural convection of Bingham fluids in a square enclosure with differentially heated side walls. J. non-Newt. Fluid Mech., 165(15-16):901-913, 2010.

[4] I. Karimfazli and I.A. Frigaard. Flow, onset and stability: Qualitative analysis of yield stress of fluid flow in enclosures. Journal of NonNewtonian Fluid Mechanics, 238:224-232, 2016.

[5] Z. Kebiche, C. Castelain, and T. Burghelea. Experimental investigation of the Rayleigh-benard convection in a yield stress fluid. Journal of Non-Newtonian Fluid Mechanics,203:9-23, 2014.

[6] A. Davaille, B. Gueslin, A. Massmeyer, and E. Di Giuseppe. Thermal instabilities in a yield stress fluid: existence and morphology. Journal of Non-Newtonian Fluid Mechanics, 193:144-153, 201.

[7] W. Thielicke, and E. J. Stamhuis. "PIVlab - Towards User-Friendly, Affordable and Accurate Digital Particle Image Velocimetry in MATLAB." Journal of Open Research Software, vol. 2, Ubiquity Press, Ltd., Oct. 2014, doi:10.5334/jors.bl. 